\title{
Massive Stars in the Local Group: Star Formation and Stellar Evolution
}

\author{
By Philip Massey ${ }^{1}$ \\ ${ }^{1}$ Lowell Observatory, 1400 W. Mars Hill Rd., Flagstaff, AZ 86001, USA
}

\begin{abstract}
The galaxies of the Local Group that are currently forming stars can serve as our laboratories for understanding star formation and the evolution of massive stars. In this talk I will summarize what I think we've learned about these topics over the past few decades of research, and briefly mention what I think needs to happen next.
\end{abstract}

\section{Introduction}

My talk today will be restricted to giving a brief introduction to the study of massive stars in the Local Group; I'll begin by discussing why I think the subject is important, and giving you a few of the complications and caveats. I'll spend most of my time then talking about what I think we've learned, first about star formation (stories of star formation, the initial mass function, and the upper mass cut-off) and second about the evolution of massive stars (including Luminous Blue Variables, Wolf-Rayet stars, and red supergaints). Finally I'll conclude with a brief discussion of where I think we need to do next. This talk is based in large part on an Annual Reviews of Astronomy 85 Astrophysics paper that I have coming out in October (Massey 2003), and the reader is referred there for a more in depth analysis. I have used this opportunity to update some of the figures and thoughts from that, so hopefully the two will be somewhat complementary.

Massive stars are extremely rare. If we integrate a Salpeter (1955) initial mass function (IMF) and allow for the relative lifetimes involved, we expect that there are about a hundred thousand solar-type stars (i.e., stars of $1( \pm 0.1) \mathcal{M}_{\odot}$ for every $20( \pm 0.2) \mathcal{M}_{\odot}$ in the Galaxy. There should be over a million solar-type stars for every $100( \pm 10) \mathcal{M}_{\odot}$ star. Yet, these stars have a disproportionate effect upon their environment: they provide most of the mechanical energy input into the interstellar medium through stellar winds and supernova (Abbott 1982); they provide most of the UV ionizing radiation of galaxies, plus power the far-IR luminosities of galaxies through the heating of dust; and they serve as the primary source of CNO enrichment of the interstellar medium (Maeder 1981).

Why study massive stars in the more distant galaxies of the Local Group, when there are so examples closer to home? The primary reason is that the metallicity of the gas out of which these stars are born differs by a factor of nearly 20 amongst the galaxies currently actively forming stars (Massey 2003 and references therein). And metallicity should matter! First, we expect that the initial mass function will depend upon metallicity, as the Jeans length depends upon temperature, and the temperature depends upon the metallicity of the star-forming cloud. Understanding how star-formation processes (and the IMF) varies with metallicity is important for interpreting the integrated properties of galaxies at large look-back times. Secondly, the stellar winds of massive stars are driven by radiation pressure in highly ionized metal lines, and the evolution of massive stars is dominated by the effects of radiatively-driven stellar winds. Thus we expect to see large differences in the evolved massive star populations depending upon the metallicity of the host galaxy.

And this is a wonderful time for such studies. Increasing sophisticated stellar evolutionary models are available through the kindness of both the Geneva and Padova teams. 
At the same time we have excellent observational capabilities, such as the high resolution optical imaging and UV spectroscopic facilities of HST. There are large-format CCD cameras available on 4-m class telescopes around the world, and my own "Local Group Survey" team is busy producing $U B V R I$ photometry of 300 million stars in 9 nearby galaxies. At the same time there are high through-put spectrographs on large telescopes, such as the Blue Channel on the MMT, GMOS on Gemini, and DEIMOS on Keck.

\subsection{Some Difficulties and Caveats}

Because massive stars have very high effective temperatures $\left(30,000-50,000{ }^{\circ} \mathrm{K}\right)$ while on the main-sequence, there are some unique problems in characterizing the massive star population of a nearby galaxy. Only a small fraction $(<10 \%)$ of their light leaks out into the visible range, and because what we see is so far down on the tail of the RayleighJeans distribution that we may not recognize the actual beast (Figure 1). The bolometric luminosity depends critically on the effective temperature, and the deduced mass depends critically on the bolometric luminosity. Massey (1998a) gives explicit equations, namely:

$$
\begin{gathered}
L \propto m^{2.0} \\
\Delta \log m=-0.2 \times \Delta M_{\text {bol }} \\
\Delta \mathrm{BC}=-6.84 \times \Delta \log T_{\text {eff }} .
\end{gathered}
$$

A reddening-free index, such as $Q$, will have a dependence $\Delta \mathrm{BC} / \Delta Q=33$ (note that the ratio is mistakenly inverted in Massey 1998a); i.e., a 0.10 mag uncertainty in $Q$ will lead to an uncertainty in the $\mathrm{BC}$ (and $M_{\mathrm{bol}}$ of $3.3 \mathrm{mag}$ and hence to an uncertainty of $0.7 \mathrm{dex}$ in $\log m$. This is not going to lead to finding a meaningful IMF slope.

Another implication of the high effective temperatures, and corresponding large bolometric corrections, is that the visually brightest stars are not the most massive. A young $85 \mathcal{M}_{\odot}$ star O-type star will be about 3 mags (a factor of 15 ) fainter than a $25 \mathcal{M}_{\odot} \mathrm{A}$-type supergiant, although the later is about $1.5 \mathrm{mag}$ (factor of 4) less luminous bolometrically. The reader is referred to Figure 1 in Massey et al. (1995) and the corresponding discussion.

In both of these cases spectroscopy provides the answer to such ambiguities, allowing accurate temperatures (and hence BCs, luminosities, and thus masses) to be determined, and allowing the construction of meaningful H-R diagrams.

\subsection{Tests}

If indeed the Local Group is our "astrophysical laboratory", then we must be good scientists and design our experiments with some care. Generally there are two types of tests possible.

(a) Mixed-age tests, where we compare the relative number of one thing to another, such as the relative number of red supergiants (RSGs) to Wolf-Rayet stars (WRs). The implicit assumption in such tests - not often stated, and possibly not always realized - is that we are averaging over a sample population to be have a completely heterogeneous mix of ages. If this assumption is invalid - if we are instead looking at a population with a strong bias of $10 \mathrm{Myr}$ (typical for a RSG) but for which 4 Myr old stars (typical for WRs) are under-represented, then we are going to have some problems in interpreting the results.

(b) Coeval tests, where we study the main-sequence stars in a cluster in order to derive a stellar IMF or determine the turn-off mass in a cluster containing evolved massive stars. We must test if indeed all the stars were "born on a particular Tuesday" (Hillenbrand etal. 1993) or if instead the age spread is significant. 
PROBLEMS WITH CONTINUUM MEASURES

(with apologies to H.J.G.L.M.L.)

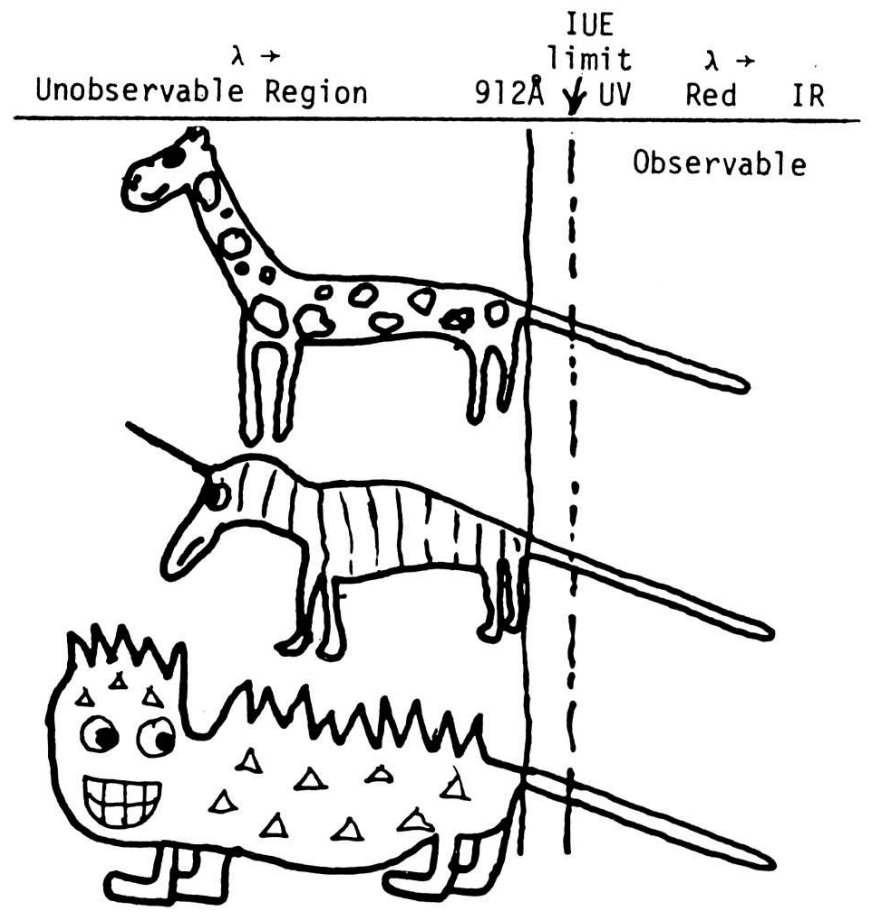

FiguRE 1. For stars with high effective temperatures, the tails of the Rayleigh-Jeans distribution gives little information about the effective temperatures, and hence the bolometric luminosities or masses of hot stars. The figure is from Conti (1986) and is used with permission.

\section{What Have We Learned?}

\subsection{Star Formation, the IMF, and the Upper-Mass Limit}

\subsubsection{Star Formation}

Detailed spectroscopic and photometric studies of young clusters reveal very different histories of star formation. Herbig (1962) first suggested that low- and intermediate-mass stars might form over a prolonged time in a cluster, followed by the formation of high mass stars, which halts all star formation. But not all clusters follow this paradigm.

Back before HST made M16 famous, Hillenbrand et al. (1993) studied the stellar content of the cluster and found that there were intermediate-mass stars pre main-sequence stars with ages as young as a few times $10^{5}$ years. Yet M16's massive star population has an age of two million years. As Hillenbrand et al. (1993) conclude, "...Thus the formation of $\mathrm{O}$ stars neither ushered in nor concluded the star-formation process in this young complex.

By contrast, the prototype "super star cluster" R136 in the 30 Doradus region of the LMC does fit the standard model. HST photometry and spectroscopy reveals that the intermediate-mass population began forming $6 \mathrm{Myr}$ ago, and stopped about $2 \mathrm{Myr}$ years 


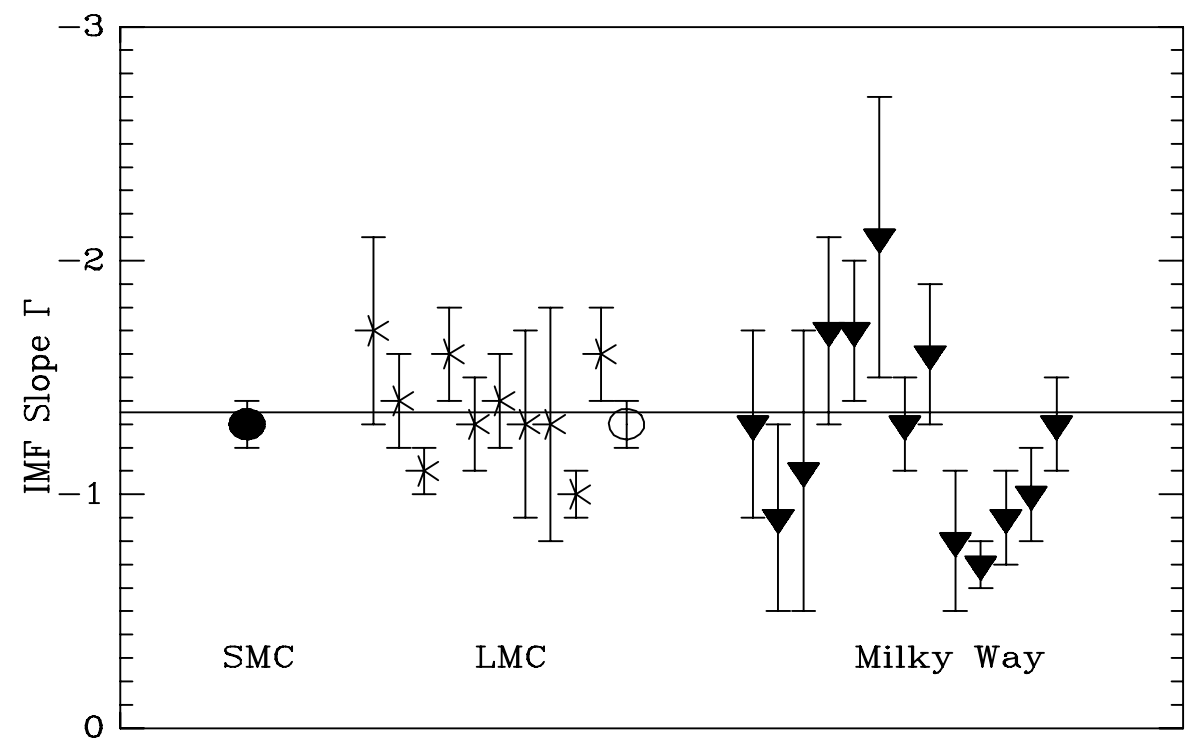

Figure 2. The slope $(\Gamma)$ of the initial mass function of OB associations and clusters in the SMC, LMC, and Milky Way is constant over a range of 4 in metallicity and 200 in stellar density. The circle shows the IMF of the R136 cluster in the LMC. The Salpeter (1955) $\Gamma=-1.35$ value is denoted by the solid line. Based upon data in Massey (2003) and references therein.

ago. This cluster contains a very large number of extremely massive stars that formed 1-2 Myr ago (Massey \& Hunter 1998).

These two clusters provide an interesting contrast in their stories of star formation. In very rich, dense clusters, such as R136, we find that the formation of intermediate-mass stars is stopped shortly after the formation of the high-mass stars, due presumably to the effects that their stellar winds have on the surrounding gas. In a less rich cluster such as M16, production of intermediate-mass stars is not halted by the formation of the high-mass stars. One might speculate that there the intermediate-mass stars will continue to form until the first Wolf-Rayet stars (with their strong stellar winds) are produced, or possibly will continue until the first SNe. Nevertheless, in both M16 and R136 the massive stars themselves were formed over a very short period of time $(<1 \mathrm{Myr})$, a fact that can prove very useful, as we'll see in Section 2.3

\subsubsection{The IMF of Massive Stars}

Studies of the stellar IMF of OB associations and clusters in the Milky Way and Magellanic Clouds show no evidence for any effect with metallicity. The variations that are seen are observational and/or statistical in nature (Massey 1998a, Kroupa 2001). In Figure 2 we see that the slope if the IMF, $\Gamma$, is approximately Salpeter $(\Gamma=-1.35)$ over a factor of 4 in metallicity and 200 in stellar density.

This is actually somewhat surprising given that metals provide the primary cooling mechanism in molecular clouds, and hence cloud temperatures (and thus the Jeans mass) should depend upon metallicity. But so far we have probed only a small range in metallicity: from the SMC to the Milky Way, the metal content changes by only a factor of 3.7. We could extend this to cover a range of 17 if we were to push such studies further out in the Local Group (WLM to Andromeda). Our Local Group survey project is pro- 
ducing $U B V R I$ photometry of 300 million stars, but will require spectroscopy if we are to determine IMFs and star formation histories.

\subsubsection{The Upper Mass Limit}

One of the other important lessons that spectroscopy of the R136 stars taught us (Massey \& Hunter 1998) is concerns the "upper mass limit" seen in clusters. The highest mass stars in R136 were an unprecedented $150 \mathcal{M}_{\odot}$. However, this is just what we would have expected from extrapolating the IMF, as this is just where the IMF would peter down to a single star. This suggests that the "upper mass limits" we've so far encountered are statistical, and not physical. Whatever it is that limits the ultimate mass of a star, we have yet to encounter it in nature.

\subsection{Evolved Massive Stars}

The evolved (He-burning) massive stars include:

- Luminous Blue Variables (LBVs): very luminous stars that undergo occasional "eruptions" (S Doradus, $\eta$ Carinae, the Hubble-Sandage variables).

- Wolf-Rayet stars (WRs): stars with broad emission lines whose surface compositions are consistent with the equilibrium products of H-burning (WN types) or He-burning (WC types).

- Red supergiants (RSGs).

\subsubsection{The Conti Scenario}

Conti (1976) first proposed that stellar winds (mass-loss) would result in some of the chemical peculiarities observed for Wolf-Rayet stars. The most luminous stars (at a given metallicity) will have the highest mass-loss rates and suffer the greatest fraction of mass loss. A modern version of the "Conti scenario" might look something like this:

$$
\begin{gathered}
m>85 \mathcal{M}_{\odot}: \mathrm{O} \longrightarrow \mathrm{LBV} \longrightarrow \mathrm{WN} \longrightarrow \mathrm{WC} \longrightarrow \mathrm{SN} \\
40>m>85 \mathcal{M}_{\odot}: \mathrm{O} \longrightarrow \mathrm{WN} \longrightarrow \mathrm{WC} \longrightarrow \mathrm{SN} \\
25>m>40 \mathcal{M}_{\odot}: \mathrm{O} \longrightarrow \mathrm{RSG} \longrightarrow \mathrm{WN} \longrightarrow \mathrm{WC} \longrightarrow \mathrm{SN} \\
20>m>25 \mathcal{M}_{\odot}: \mathrm{O} \longrightarrow \mathrm{RSG} \longrightarrow \mathrm{WN} \longrightarrow \mathrm{SN} \\
10>m>20 \mathcal{M}_{\odot}: \mathrm{OB} \longrightarrow \mathrm{RSG} \longrightarrow \mathrm{BSG} \longrightarrow \mathrm{SN}
\end{gathered}
$$

Thus, an $85 \mathcal{M}_{\odot}$ star starts out as an O-type star, and then becomes a luminous blue variable. Extreme mass-loss during the LBV stage then helps lead first to a WN-type Wolf-Rayet star (with the H-burning products revealed at the surface), while subsequent evolution (and mass-loss) results in a WC-type WR (with the more advanced He-burning products revealed at the surface. Stars of lower mass $\left(20-25 \mathcal{M}_{\odot}\right)$ might not have enough mass loss for a star to evolve beyond the WN stage, while stars of even lower mass might never go through a WR phase at all, but rather spend their He-burning lives first as red supergiant (RSG) and then as a "second generation" blue supergiant (BSG), similar to the precursor of SN1987A.

Of course, we don't know how right this overall "cartoon" version of massive star evolution is, and in particular we don't know what the corresponding mass ranges are for the various evolutionary paths. We especially don't know how these ranges vary with metallicity!

\subsection{2. $L B V s$}

Hubble \& Sandage (1953) called attention to five irregular variables in M31 and M33 that were (at times) among the brightest resolved stellar objects in these galaxies. Pho-

tographic plates from Mt. Wilson extended back to 1916, which revealed episodic visual brightenings of several magnitudes. These objects were extremely luminous and blue, 
and had spectra that were of intermediate F-type during maxima. A footnote in their paper suggested that the LMC star S Doradus might be similar. The connection to the Galactic stars $\eta$ Car and P Cyg came later. Conti (1984) coined the term "luminous blue variables" to describe these objects.

LBVs are extremely luminous bolometrically, and are found near the edge of the observed upper luminosity limit in the H-R diagram. They undergo episodic bouts of high mass loss, during which they brighten visually, while remaining roughly constant in bolometric luminosity.

In a series of papers in the late 1970s and early 1980s, Roberta Humphreys demonstrated that there was an observed limit to the luminosities of stars (see, for example, Humphreys \& Davidson 1979). This limit decreases with decreasing effective temperatures until $T_{\text {eff }}=10,000^{\circ} \mathrm{K}$, after which it is nearly constant at $/ \log L / L_{\odot} \sim 5.7$ $\left(M_{\text {bol }}=-9.5\right.$, corresponding roughly to $50 \mathcal{M}_{\odot}$. It was understood that this upper luminosity limit was somehow related to LBVs, since these star occupied a narrow band near this limit.

It was very tempting to try to interpret the observed upper luminosity limit in terms of some fundamental physics, such as the Eddington limit. In the classic Eddington limit,

$$
\frac{L}{M}=\frac{4 \pi G c}{\kappa}
$$

where $\kappa$ is the flux-mean opacity ( 0.347 for electron scattering if $T_{\text {eff }}$ is high). In that case

$$
\frac{L / L \odot}{M / M \odot}=3.8 \times 10^{4}
$$

This is similar to, but considerably greater than, the observed $\frac{L / L_{\odot}}{M / M_{\odot}}$ ratio of the HumphreysDavidson limit:

$$
\frac{10^{5.7} L_{\odot}}{50 \mathcal{M}_{\odot}}=1.0 \times 10^{4}
$$

This is tantalizingly close, but the classical Eddington limit is thus factors of several times higher than what is observed, and doesn't show the same $T_{\text {eff }}$ dependence.

Lamers \& Fitzpatrick (1988) used model atmospheres to explore where radiation pressure and gravity are balanced if the full effects of metal-line opacities are included, rather than just the effects of electron scattering. This turned out to completely explain the Humphreys-Davidson limit. The actual limit is a trough, descending to lower luminosity with decreasing effective temperatures until a temperature of $10,000^{\circ} \mathrm{K}$ is reached, after which the opacity decreases and hence the allowed luminosity increases again. However, since stars evolve from higher temperatures to lower, the effect is to produce a decreasing envelope followed by a line at constant luminosity luminosity for $T_{\mathrm{eff}}<10,000^{\circ} \mathrm{K}$.

This suggests then that LBVs are a normal phase in the lives of the most massive stars. As such stars try to evolve to cooler effective temperatures, their radii expand, their surface gravities get lower, and most importantly their atmospheric opacities increase and radiation pressure overcomes gravity, leading to vastly increased mass loss. These stars are stopped dead in their (evolutionary) tracks! Such a star will dump a lot of material, heat up, and then try again.

This may neatly wrap up the whole issue of LBVs, but possibly not. Kenyon \& Gallagher (1985) suggested that some LBVs were the result of evolution in close binary systems, an argument partially supported by the relative isolation of some LBVs in M31. This has been given some boast by the controversial claim that $\eta$ Car itself is a binary.

The problem with fully resolving the question of the origins of LBVs is complicated 


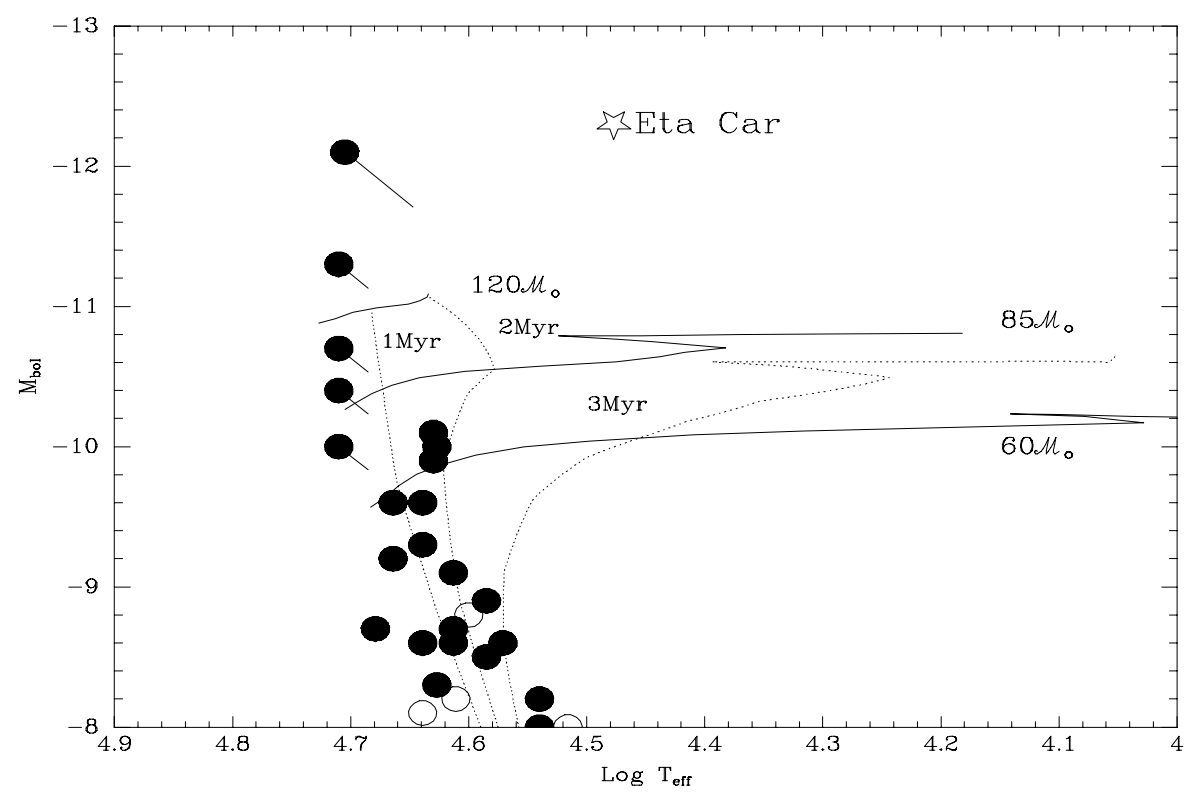

Figure 3. The location of $\eta$ Car in the H-R diagram is just what we expect if it happens to be a highly evolved massive star, as its bolometric luminosity is slightly higher than the highest luminosity unevolved massive star. The highest mass stars are quite coeval, with an age of about 1 Myr. This figure is based upon Figure 4 in Massey, DeGioia-Eastwood, \& Waterhouse (2001).

by the fact that the bolometric luminoisities of LBVs are poorly known, making their placement on the H-R diagram uncertain. The real exception to this is $\eta$ Car itself, where the surrounding gas and dust reprocesses the radiation to the IR, where it can be measured. $\eta$ Car is a member of the Tr14/16 complex, and when we place it on the H-R diagram (Figure 3) we find something quite comforting: it is exactly where it should be, in the sense of being just slightly more bolometrically luminous than the highest luminosity unevolved star (Massey, DeGioia-Eastwood, \& Waterhouse 2001). One is forced to conclude that even if $\eta$ Car is a binary, its binary nature may be irrelevant to its LBV nature.

Another complication in understanding the origins of LBVs is that it is very hard to gather meaningful statistics. $\eta$ Car underwent its last major ourburst in 1830, and P Cyg underwent its last major outburst in 1655. If these stars were located in the Magellanic Clouds, would we even consider them LBVs? One such Galactic example of an LBV-inwaiting may be the star VI Cyg No. 12 (Massey \& Thompson 1991). This star is one of the visually most luminous stars known in the Milky Way, it is surrounded by circumstellar material, but it has not had a photometric "episode" during the few decades its been known. Given that Hubble \& Sandage (1953) relied upon 40 years worth of observations just to identify the first 5 such stars in M31 and M33 (one of these 5 stars, Var A, is no longer even considered an LBV), it may require centuries to obtain firm statistics on the number of LBVs in nearby galaxies.

\subsubsection{Wolf-Rayet Stars}

In 1867 two French astronomers, Wolf and Rayet, came across three stars whose spectra showed broad, strong emission lines rather than the absorption spectra that character- 
ized other stars. Beals (1930) correctly identified the emitting atoms as ionized helium, nitrogen, and carbon.

WN-type Wolf-Rayets show lines of helium and nitrogen. Abundance studies show that for WN stars we are seeing an atmospheric composition that reflects what the composition that the stellar core would have during the main-sequence CNO hydrogen-burning cycle. (See discussion and references in Massey 2003.) WC stars show helium, carbon, and oxygen; the latter two are the products of the triple- $\alpha$ helium-burning cycle. Somehow, then, the outer layers of these stars have been stripped away, revealing the nuclear burning products at the surface. Prior to 1976, the prevailing notion was that Wolf-Rayet stars were the product of evolution in close binaries, with Roche-lobe overflow being responsible for this stripping. However, Conti (1976) noted that the ubiquitous presence of stellar winds in hot stars provided an alternative explanation, and proposed that O-type stars would naturally evolve first to WN-type and then to WC-type Wolf-Rayets.

Since mass-loss depends upon metallicity, one expects that the relative number of WCs and WNs should vary from place to place. Indeed, roughly equal number of WCs and WNs were known in the vicinity of the sun, while in the lower metallicity Magellanic Clouds most of the WRs are of WN type. quantitative numbers provide the means for comparison with evolutionary models.

One complication in finding unbiased samples of Wolf-Rayet stars is that the WCs are much easier to find than are the WNs. The strongest emission line in WCs (CIII $\lambda 4650$ ) have a median line strength that is a factor of 4 grater than that of the strongest emission line in WNs (HeII $\lambda 4686$ ). My colleagues and I designed a set of 3 interference filters in order to facilitate identification of WRs in nearby galaxies, and have used this with some success in regions of nearby galaxies (see Massey \& Johnson 1998 for a recent summary of such studies). Candidate stars are identified on the basis of having a magnitude difference from an emission-band to continuum-band filter, if that magnitude difference is significant compared to the photometric errors. Candidates are then observed spectroscopically.

Such studies have revealed a strong trend in the relative number of WCs and WNs with respect to metallicity. We show the data in Figure 4 Two points deserve comments. First, the point for the Milky Way is higher than the trend based upon the other galaxies, and one has to wonder if the data for the region within $2 \mathrm{kpc}$ is complete. No systemic surveys for WRs in the Milky Way have been carried out; instead, such stars were found either as part of the HD survey (which would be very incomplete at a distance of $2 \mathrm{kpc}$ for WRs given typical reddenings, as noted by Massey \& Johnson 1998), or by "accidental" spectroscopic discovery as part of a study of stars in a specific cluster, say. IC10 poses a far more interesting discrepancy. Massey \& Armandroff (1995) discovered 22 Wolf-Rayet candidates in this small galaxy (by contrast, the SMC, which is twice the size, contains only 11 known WRs), and argued that IC10 is the nearest example of a starburst galaxy. One peculiarity though is that the number of WCs was about 2 times greater than the number of WNs, while a ratio of 0.1 would be more in keeping with its metallicity. Various explanations have been advanced to explain this: possible the IMF is top-heavy, or alternatively massive stars might have formed within the same short time interval all across the galaxy and are evolving in lock-step. A recent deeper survey by Massey \& Holmes (2002) suggests another explanation: they found a large number of additional candidates, confirming two by spectroscopy. If spectroscopic confirmation of the remainder turns out the way they expect, then the number rati of WCs to WNs may be normal in IC 10, but the galaxy may have a total WR content of a $100 \mathrm{WRs}$, a surface density that would be about 20 times higher than that of the LMC. In terms of its overall massive star population, IC10 must be more like an OB association, but on a kpc scale. 

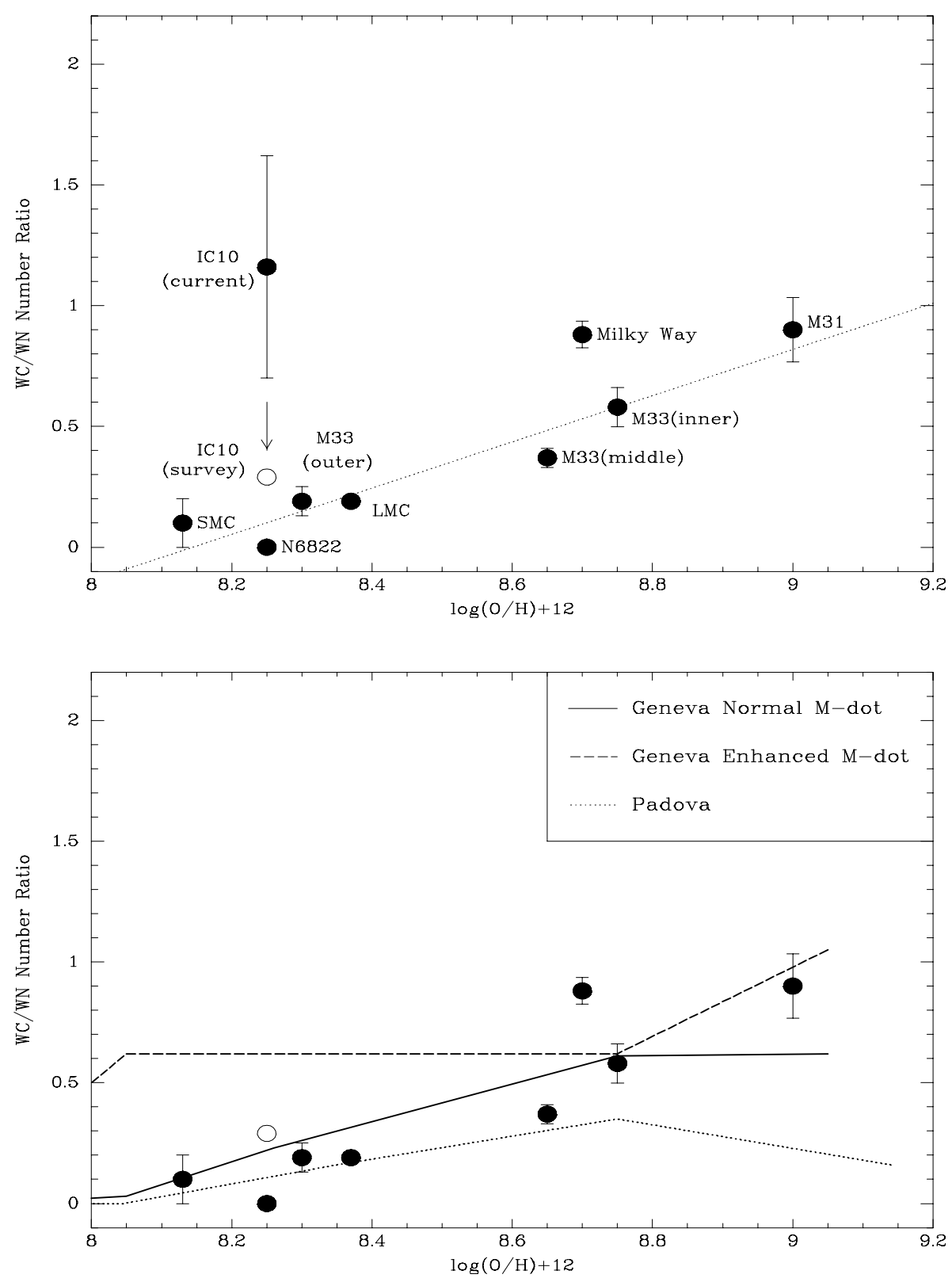

FiguRE 4. The relative number of WCs and WNs show a strong trend with metallicity among the Local Group galaxies (top). The normal-mass loss models of the Geneva and Padova groups do a reasonable job at matching the data for all but the highest metallicities (bottom). The "enhanced" mass-loss models of the Geneva group do not match the data at all.

How well do the stellar models do in reproducing this trend? We see in the bottom panel of Figure 4 that the normal-mass loss models of the Geneva (Schaller et al. 1992; Schaerer et al. 1993; Charbonnel et al. 1993) and Padova (Fagotto et al. 1994; Bressan et al. 1993) groups come relatively close to reproducing the trends, although both predict too low a WC/WN at higher metallicities. (One should note, though, that the statistics 
for M31 are not well established.) The "enhanced" mass-loss tracks of the Geneva group (Meynet et al. 1994) do not match the data at all. Massey (2003) has examined the historical basis for the introduction of these "enhanced" mass-loss models, and with the advantage of 20-20 hindsight suggests that the problems these were trying to solve were to some extent unreal. Unfortunately, it is the enhanced mass-loss models that are commonly used in starburst models (e.g., Schaerer \& Vacca 1998; Leitherer et al. 1999; Smith, Norris, \& Crowther 2003).

\subsubsection{Red Supergiants}

A long-standing problem in identifying the RSG content of nearby galaxies has been the confusion by foreground Galactic dwarfs. For instance, Massey (1998b) found that roughly half of the sample of red stars seen towards M33 by Humphreys \& Sandage (1980) were likely foreground Galactic objects. $B V R$ two-color diagrams partially resolve this ambiguity, as RSGs will have redder $B$ - $V$ colors at a given $V$ - $R$ color (Massey 1998b). Spectroscopy of the Ca II triplet lines in the NIR is a powerful way of distinguishing Galactic disk dwarfs from bona-fide extra-galactic RSGs given the heliocentric radial velocities of most Local Group galaxies.

Massey (1998b) investigated the RSG content of three Local Group galaxies (NGC 6822, M33, and M31), which span a range of 0.8 dex in metallicity. He found that as the metallicity goes up, the fraction of high luminosity RSGs goes down, but that there is not a sharp cut-off in either $M_{V}$ or $M_{\text {bol }}$. This suggests that moderately high-mass stars $\left(40 \mathcal{M}_{\odot}\right)$ become RSGs even at higher metallicities, but what changes is that the RSG phase is shorter at higher metallicities, in accord with the suggestion of Maeder, Lequeuex, \& Azzopardi (1980). A recent study (Massey \& Olsen 2003) has extended this work to that of the Magellanic Clouds.

This work has revealed a potentially serious drawback to stellar evolutionary models, namely that none of the "modern" stellar evolutionary models produce RSGs as cool and luminous as what is actually observed. This is illustrated in Figure 5 We see too that there is a tight relationship between luminosity and effective temperature of the RSGs. Matching this will provide a challenge to future stellar evolutionary models.

Finally we note that the relative number of RSGs and WRs show a very strong trend with metallicity, changing by two orders of magnitude over just 0.8 dex in metallicity (Figure [6] Again, none of the stellar evolutionary models reproduce this trend.

\subsection{Coeval Tests}

So far the tests described have involved mixed-aged populations, where we compare the relative number of WCs to WNs, or RSGs to WRs. But, in principle a far more sensitive test is possible, which involves using coeval regions to determine the progenitor masses as a function of metallicity.

This is a classical method first used by Sandage (1953) to determine the masses of RR Lyrae stars using globular clusters, and subsequently used by Anthony-Twarog (1982) to find the progenitor masses of white dwarfs in intermediate-age clusters. However, it is one thing do do this for clusters that are $10^{10} \mathrm{yr}$ old, or even 40-70 Myr old, and quite another to do it for clusters that are only a few million years old: the degree of coevality required is quite high. However, recall that Hillenbrand et al. (1993) study of NGC 6611 (discussed in Section 2.1.1) found that most of the massive stars were born over a very short period of time ( $<1 \mathrm{Myr})$. The data used to construct the $\mathrm{H}-\mathrm{R}$ diagrams themselves can be used to answer the degree of coevality.

Massey, Waterhouse, \& DeGioia-Eastwood (2000) and Massey, DeGioia-Eastwood, \& Waterhouse (2001) selected 19 clusters in the Magellanic Clouds and 12 clusters in the 

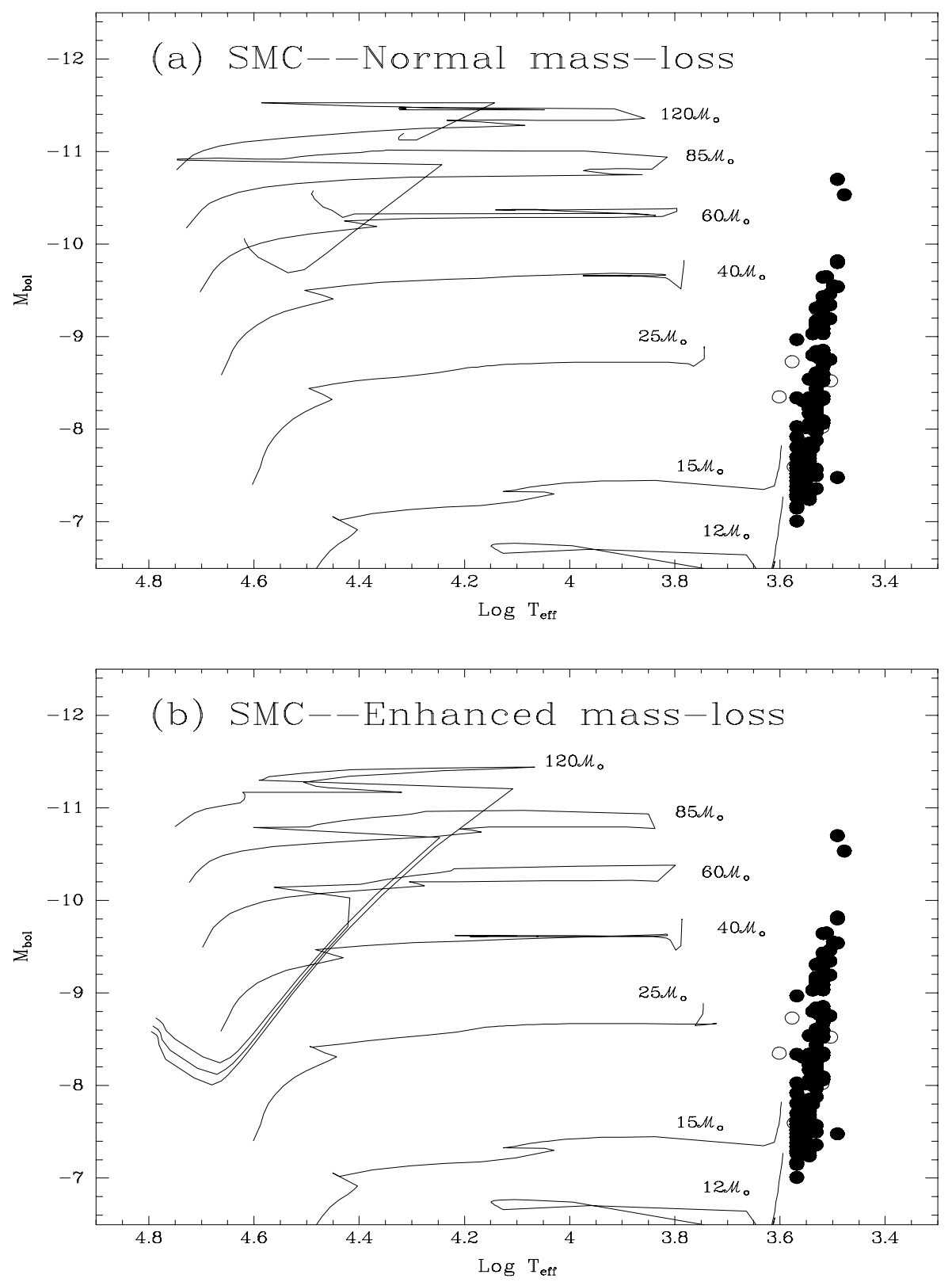

FiguRE 5. The evolutionary tracks do not actually go sufficiently far to the right to produce RSGs as cool and as luminous as what is observed. Note too that the tight relation of RSGs in these diagrams, with stars of lower luminosities being of slightly earlier (hotter) spectral types. The figure is based upon data from Massey \& Olsen (2003).

Milky Way that contained evolved massive stars, and obtained photometry and spectroscopy of the most luminous unevolved cluster members. About half of these met their stringent criteria for coevality, and hence could be used to determine the progenitor masses of the evolved massive stars using the masses of as-yet unevolved massive stars. The results are shown in Figure 7 


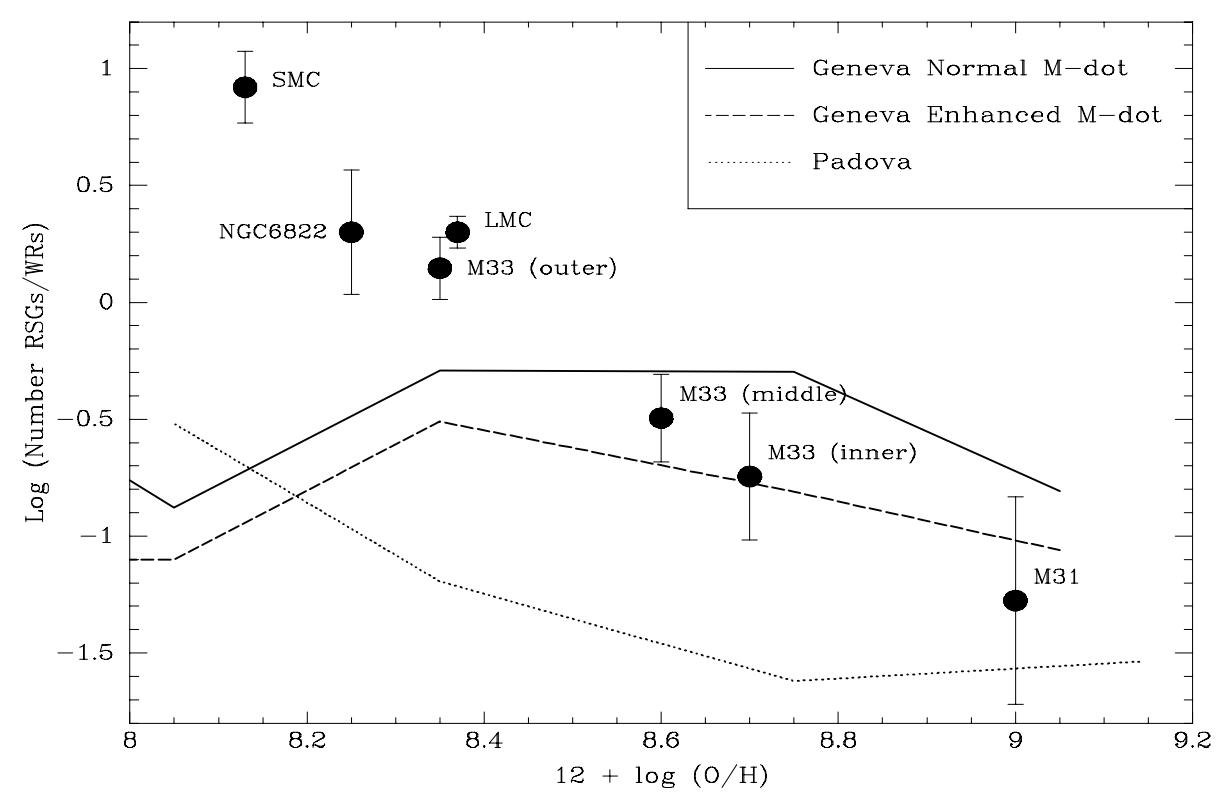

FiguRE 6. Although the relative number of RSGs and WRs show a very strong trend with metallicity, none of the current (non-rotation) evolutionary models reproduce this trend.

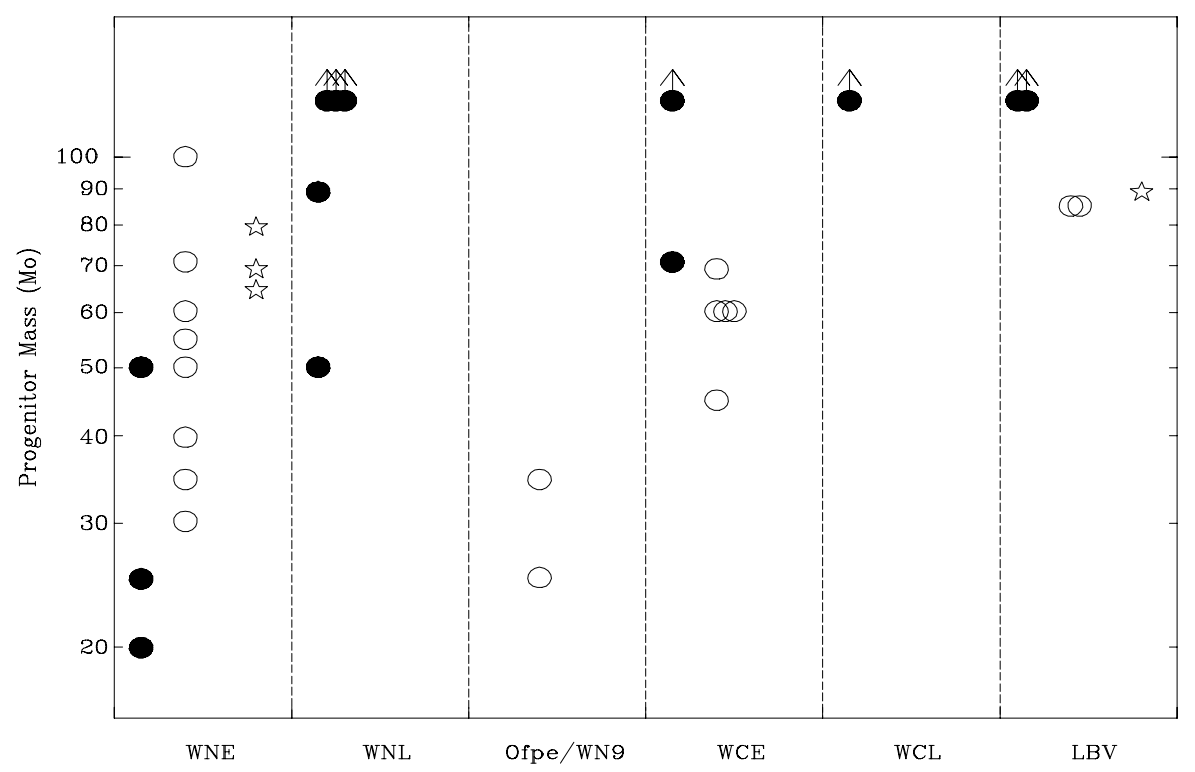

FiguRE 7 . The progenitor masses are shown for various types of evolved massive stars for the SMC (stars), LMC (open circles), and Milky Way (filled circles). We see that LBVs come from only the most massive stars. The Ofpe/WN9s come from lower mass stars. Of the WNEs, the progenitor masses are highest in the SMC, span a larger range to lower masses in the LMC, and are even lower in the Milky Way, as one might expect. The figure is is modified from one that appears in Massey et al. (2001). 
What we've learned from this is that LBVs all come from the highest mass stars, as we expect. The Ofpe/WN9s, which have sometimes been linked to the LBVs, do not come from the highest mass stars - instead, their progenitor masses are quite low. Interestingly, are strong trend in the progenitor masses of WN stars in the three galaxies: in the SMC only the highest mass stars become WRs, apparently, which is consistent with the SMC's low metallicity and the expectations of the Conti scenario that the amount of mass-loss will determine the evolution of massive stars. In the LMC, a larger range of progenitor masses yield WNs. And, in the Milky Way, stars from masses as low as $20 \mathcal{M}_{\odot}$, up to that of $>>120 \mathcal{M}_{\odot}$ become WRs.

\section{What's Next?}

Studies of the massive star content of nearby galaxies (including our own!) have shed important light on star-formation and the evolution of massive stars. What comes next?

- We need to move beyond the Magellanic Cloud st complete galaxy-wide surveys for WRs, RSGs, and BSGs in M31, M33, NGC 6822, IC10, and other galaxies of the Local Group.

- Follow-up spectroscopy with 8-m class telescopes will allow meaningful H-R diagrams to be constructed, allowing careful tests of models as a function of metallicity.

- Coeval studies need to be extended to higher metallicity systems, such as M31

This contribution has been supported by the National Science Foundation, through grant AST0093060.

\section{REFERENCES}

Abbott, D. C. 1982, ApJ 163, 723

Anthony-Twarog, B. J. 1982 ApJ, 255, 245

Beals, C. S. 1930, Publ. Dominion Astrophys. Obs., 4, 271

Bressan, A., Fagotto, F., Bertelli, G., \& Chiosi, C. 1993, A\&AS, 100, 647

Charbonnel, C., Meynet, G., Maeder, A., Schaller, G., \& Schaerer, D. 1993, A\&AS, 101, 415

Conti, P. S. 1976, Mem. Soc. R. Sci. Liege 9, 193

Conti, P. S. 1984, in Observational Tests of the Stellar Evolutionary Theory, IAU Symp. 105, ed. A. Maeder \& A. Renzini (Dordrecht: Reidel), 253

Conti, P. S. 1986, in Luminous Stars and Associations in Galaxies, IAU Symp. 116, ed. C. W. H. de Loore, A. J. Willis, \& P. Laskarides (Dordrecht: Reidel), 199

Fagotto, F., Bressan, A., Bertelli, G., Chiosi, C. 1994, A\&AS, 105, 29

Herbig, G. H. 1962, ApJ, 135, 736

Hillenbrand, L. A., Massey, P., Strom, S. E., \& Merrill, M. K. 1993, AJ, 106, 1906

Hubble, E., \& Sandage, A. 1953, ApJ, 118, 353

Humphreys, R. M., \& Davidson, K. 1979, ApJ, 232, 409

Humphreys, R. M., \& Sandage, A. 1980, ApJS, 44, 319

Kenyon, S. J., \& Gallagher, J. S. 1985, ApJ, 290, 542

Kroupa. P. 2001, MNRAS, 322, 231

Lamers, H. J. G. L. M., \& Fitzpatrick, E. L. 1988, ApJ, 324, 279

Leitherer, C., Schaerer, D., Goldader, J. D., Delgado, R. M. G., Robert, C., et al. 1999, ApJS, 123,3

Maeder, A. 1981, A\&A 101, 385

Maeder, A., Lequeuex, J., \& Azzopardi, M. 1980, A\&A, 90, L17

Massey, P. 1998a, in The Stellar Initial Mass Function, 38th Herstmonceux Conference, ed. G. Gilmore \& D. Howell (San Francisco: ASP), 17

Massey, P. 1998b, ApJ, 501, 153

Massey, P. 2003, ARA\&A, 41, 15 
Massey, P., \& Armandroff, T. E. 1995, AJ, 109, 2470

Massey, P., DeGioia-Eastwood, K., \& Waterhouse, E. 2001, AJ, 121, 1050

Massey, P., \& Holmes, S. 2002, ApJ, 580, L35

Massey, P., \& Hunter, D. A. 1998, ApJ, 493, 180

Massey, P., \& Johnson, O. 1998, ApJ, 505, 793

Massey, P., Lang, C. D., DeGioia-Eastwood, K., \& Garmany, C. D. 1995, ApJ, 438, 188

Massey, P., \& Olsen, K. A. G. 2003, AJ, submitted

Massey, P., \& Thompson, A. B. 1991, AJ, 101, 1408

Massey, P., Waterhouse. E., \& DeGioia-Eastwood, K. 2000, AJ, 119, 2214

Meynet, G., Maeder, A., Schaller, G., Schaerer, D., \& Charbonnel, C. 1994, A\&AS, 103, 97

Salpeter, E. E. 1955, ApJ, 121, 161

Sandage, A. 1953, Mem. Soc. R. Sci. Liege 4, 254

Schaerer, D., Meynet, G., Maeder, A., \& Schaller, G. 1993, A\&AS, 98, 523

Schaerer, D., \& Vacca, W. D. 1998, ApJ, 497, 618

Schaller, G., Schaerer, D., Maynet, G., \& Maeder, A. 1992, A\&AS, 96, 269

Smith, L. J., Norris, R. P. F., \& Crowther, P. 2002, MNRAS, 337, 1309

The Local Group as an Astrophysical Laboratory, 2003 STScI May Symposium, eds. M. Livio et al., (Cambridge: Cambridge U. Press) 